\title{
Tradução e validação para a língua portuguesa da escala de neofobia em relação à tecnologia de alimentos: food technology neophobia scale
}

\author{
Translation and validation to portuguese language of food technology neophobia scale
}

\author{
Márcia Cristina Teixeira Ribeiro Vidigal ${ }^{\mathrm{I}}$ Valéria Paula Rodrigues Minim ${ }^{\mathrm{I}}$ Rêmio Teixeira Moreira ${ }^{\mathrm{I}}$ \\ Ana Clarissa dos Santos Pires ${ }^{\mathrm{I}}$ Marco Aurélio Marques Ferreira ${ }^{\mathrm{II}}$ \\ Aline Cristina Arruda Gonçalves ${ }^{\mathrm{III}}$ Luis Antônio Minim ${ }^{\mathrm{I}}$
}

RESUMO

As indústrias de alimentos vêm inovando $e$ desenvolvendo novos produtos e processamentos, e os consumidores estão mais conscientes e preocupados em relação às novas tecnologias alimentares, o que pode influenciar a aceitação dos alimentos. Nesse contexto, objetivou-se disponibilizar para a língua portuguesa o questionário originalmente desenvolvido em inglês que avalia a neofobia em relação à tecnologia de alimentos, denominado Food Technology Neophobia Scale (FNTS). O instrumento original composto por treze afirmações foi inicialmente traduzido para o português por três indivíduos bilíngues, de forma independente; na sequência, as versões em português foram traduzidas novamente para o inglês por outros três indivíduos bilíngues, também de forma independente. Procedeu-se então à análise das versões e ajustes para se obter uma única versão em português com equivalência conceitual e linguística, a qual recebeu o nome de Escala de Neofobia em relação à Tecnologia de Alimentos. As versões original e traduzida foram aplicadas a 30 indivíduos bilíngues para avaliação da confiabilidade e reprodutibilidade do questionário. Os resultados obtidos demonstraram que a tradução do instrumento foi adequada e confiável, podendo, portanto, ser empregada em estudos com consumidores brasileiros.

Palavras-chave: aversão, nova tecnologia, validação, consumidor, escala psicométrica.

\section{ABSTRACT}

The food industries have been innovating and developing new products and processes. Consumers are aware and well concerned about new food technologies, which can influence food acceptance. In this context, the objective was to provide a portuguese version of the questionnaire developed in English to evaluate neophobia for food technology called Food Technology Neophobia Scale (FNTS). The original instrument consists of thirteen items and was initially translated into Portuguese by three bilingual individuals independently. Following the Portuguese versions were translated back into English by three other bilingual individuals, also independently. Analysis of versions and adjustments were performed to obtain a single version in Portuguese with linguistic and conceptual equivalence, which was called "Escala de Neofobia em relação à Tecnologia de Alimentos". The original and translated versions were applied to 30 bilingual individuals to evaluate the reliability and reproducibility of the questionnaire. The results showed that the translation of the instrument was adequate and reliable, therefore can be used in studies with Brazilian consumers.

Key words: aversion, new technology, validation, consumer, psychometric scale.

\section{INTRODUÇÃO}

Há alguns anos, o setor de alimentos tem sido confrontado com um mercado cada vez mais competitivo e globalizado e com uma demanda muito mais rigorosa por parte dos consumidores, que estão mais instruídos e exigentes. Esta situação obrigou as indústrias de alimentos a inovar e desenvolver novos produtos e processamentos, a fim de tornar a comercialização mais competitiva e atender às exigências dos consumidores (BARRENA \& SANCHÈZ, 2012). No entanto, mesmo que um alimento seja nutritivo e traga beneficios à saúde do consumidor, é improvável que ele seja aceito se as características sensoriais não forem agradáveis (VIDIGAL et al., 2011). Além disso, os métodos de

\footnotetext{
'Departamento de Tecnologia de Alimentos, Universidade Federal de Viçosa, (UFV), Av. Peter Henry Rolfs, s/n., 36570000, Viçosa, MG, Brasil. E-mail: vprm@ufv.br. *Autor para correspondência.

IIDepartamento de Administração, UFV, Viçosa, MG, Brasil.

IIIPrograma Institucional de Bioengenharia, Universidade Federal de São João Del-Rei (UFSJ), Sete Lagoas, MG, Brasil.
} 
produção do alimento e as etapas de processamento também são uma preocupação crescente para muitos consumidores. Vários estudos têm demonstrado que algumas inovações tecnológicas estão em debate sobre seus méritos e desvantagens em muitos países do mundo (BEHRENS et al., 2009; CARDELLO, 2003; DELIZA et al., 2003a).

Tecnologias envolvendo transgênicos, aplicadas à alimentação, muitas vezes, geram percepção de elevado risco e de aversão entre os consumidores (HANSEN et al., 2003). Para outras tecnologias alimentares, a aceitação do consumidor é ainda uma questão não definida. Como exemplo, temos a nanotecnologia, que tem um grande potencial de gerar novos produtos e processos e está cada vez mais sendo empregada no processamento de alimentos e embalagens (SANGUANSRI \& AUGUSTIN, 2006). As novas tecnologias alimentares permitem inovações no setor de alimentos. No entanto, nem todas as tecnologias são igualmente aceitas pelos consumidores, que são o destino final de todo e qualquer produto que seja desenvolvido. Dessa forma, é de fundamental importância conhecer o comportamento dos consumidores em relação a um novo produto.

Embora, em alguns países, não seja obrigatória a divulgação do tipo de tecnologia utilizada no processamento do alimento, a possibilidade de acesso à informação pelos consumidores (por exemplo, pela internet) sugere que a indústria de alimentos deva avaliar a aceitação das novas tecnologias, ao invés de arriscar uma reação negativa quando tal informação é descoberta (EVANS \& COX, 2006). Sendo assim, testar a aceitação do conceito do produto pelo consumidor antes do seu desenvolvimento é o mais sensato (van KLEEF et al., 2005).

Há evidências de que alguns consumidores estão receosos em relação aos novos alimentos e às novas tecnologias (RONTELTAP et al., 2007; BIEBERSTEIN et al., 2013). Segundo CHEN et al. (2012), para a aceitação de alimentos obtidos por tecnologias não-convencionais, torna-se necessário investimento em programas de informação e educação dos consumidores, para reduzir "os medos" e evitar o fracasso da inovação no mercado. De acordo com SIEGRIST (2008), a percepção dos benefícios, dos riscos e da naturalidade, além da confiança por parte dos consumidores e o impacto dos construtos psicológicos, como a neofobia (aversão ao novo), são os fatores mais importantes para a aceitação de novas tecnologias alimentares.

Dada a complexidade das decisões de consumo alimentar, uma das questões investigadas nos últimos anos é a rejeição de algumas pessoas em relação aos alimentos novos ou desconhecidos, um fenômeno conhecido como neofobia alimentar. Pessoas neofóbicas tendem a exibir atitudes negativas e apresentar menor expectativa em relação ao sabor dos alimentos (BARRENA \& SANCHÈZ, 2012). PLINER \& HOBDEN (1992) desenvolveram a escala de neofobia alimentar, que é uma ferramenta útil para avaliar as reações dos consumidores em relação aos alimentos novos, étnicos ou não familiares, porém é menos adequada para avaliar a receptividade aos alimentos produzidos por novas tecnologias. Pela dificuldade de estabelecer os limites de aceitação desses alimentos, COX \& EVANS (2008) desenvolveram uma nova ferramenta psicométrica que identifica neofobia em relação à tecnologia de alimentos. A capacidade de identificar segmentos da população que têm maior ou menor neofobia é útil, pois permite a identificação precoce de grupos que estão dispostos a aceitar produtos inovadores, principalmente quando a nova tecnologia pode proporcionar benefícios. O questionário Food Technology Neophobia Scale (FTNS) é composto por treze afirmações, das quais seis estão relacionadas à descrição "novas tecnologias são desnecessárias"; quatro itens à percepção de risco; dois itens à escolhas saudáveis; e um item à informação fornecida pela mídia.

Os instrumentos utilizados para avaliar o conhecimento, atitude e comportamento dos consumidores frente a diversos aspectos relacionados aos alimentos geralmente foram desenvolvidos em países cuja língua-mãe não é o português. Nesse caso, existem duas alternativas: o desenvolvimento de instrumentos no idioma desejado ou, então, a utilização daqueles já existentes, sendo necessário submetê-los às regras internacionais de tradução, adaptação cultural e validação para a língua-alvo (DANTAS et al., 2003). O processo de adaptação inclui a tradução, retrotradução (back-translation), análise de ambas as versões e aplicação do instrumento a uma amostra bilíngue da população, o que permite avaliar a equivalência entre as duas versões linguísticas do instrumento (PRIETO, 1992). Vários autores têm se preocupado em fazer a tradução para o português de questionários originalmente desenvolvidos em língua inglesa (DELIZA et al., 2003b; SOARES et al., 2006; CORSO \& BENASSI, 2012).

Opresente trabalho objetivou disponibilizar para a língua portuguesa o questionário originalmente desenvolvido em inglês, Food Technology Neophobia Scale (COX \& EVANS, 2008), para ser utilizado na avaliação da neofobia dos consumidores brasileiros em relação à tecnologia de alimentos. 


\section{MATERIAL E MÉTODOS}

\section{Instrumento}

A versão original (em inglês) do instrumento elaborado por COX \& EVANS (2008) foi desenvolvida na forma de um questionário contendo 13 itens (Tabela 1), os quais são apresentados na forma de afirmações, em que o respondente deve expressar a sua opinião utilizando escala de concordância (escala ancorada nos extremos 1 = "discordo totalmente" e 7 ="concordo totalmente").

\section{Tradução e Retrotradução}

O processo de tradução do FTNS para a língua portuguesa foi realizado por indivíduos fluentes em ambas as línguas (português e inglês). As treze questões que compõem o questionário foram traduzidas de forma independente para o português por três tradutores bilíngues, dos quais dois apresentam formação em Ciência e Tecnologia de Alimentos e um é professor de inglês. Tais versões foram traduzidas para o inglês (retrotradução) por outros três indivíduos bilíngues, tendo eles já residido em país de língua inglesa. A adaptação do questionário para a língua portuguesa foi realizada utilizando a técnica back-translation (PRIETO, 1992). As versões traduzidas foram comparadas com a versão original e os ajustes necessários foram realizados, a fim de se obter a equivalência conceitual e linguística, chegando, assim, à versão final do questionário em português.

\section{Validação do Instrumento}

Para validação e confiabilidade do questionário, indivíduos bilíngues avaliaram as propriedades psicométricas do instrumento na linguagem original e na nova linguagem. Foram recrutados para participar do estudo 30 indivíduos, sendo 25 estudantes de graduação e de pós-graduação na área de Alimentos, que leem e entendem os dois idiomas (inglês e português) e cinco professores de inglês. $\mathrm{O}$ tamanho da amostra foi baseado nas recomendações de OHRBACH et al. (2009). O grupo foi composto por 21 mulheres e nove homens, com idade variando entre 20 e 35 anos. Os indivíduos foram aleatoriamente divididos em grupos 1 e 2, sendo que o grupo 1 recebeu primeiro a versão em português e, após um intervalo de 1 a 2 semanas, conforme sugerido por PETER (1979), respondeu a versão em inglês. $O$ grupo 2 trabalhou na versão inglesa e, posteriormente, na portuguesa, obedecendo ao mesmo intervalo de tempo entre as respostas. As treze questões foram avaliadas em uma escala não estruturada ancorada nos extremos $(1=$ "discordo totalmente" e 7 ="concordo totalmente").

\section{Análise Estatística}

A reprodutibilidade entre a versão inglesa e portuguesa e a confiabilidade do questionário foram avaliadas. Para a comparação dos resultados obtidos nas duas versões, foi usado o teste de Wilcoxon para dados não paramétricos. Os testes não paramétricos não estão condicionados por qualquer distribuição de probabilidades dos dados em análise e podem ser aplicados para pequenas amostras. Neste caso, o teste de hipótese avalia se as respostas dadas para as duas versões foram idênticas, ou seja, se a média das diferenças entre as observações foi nula, considerando-se significante $\mathrm{P}<0,05$.

$$
\begin{aligned}
& \mathrm{H}_{0}: \mu_{d}=0 \\
& \mathrm{H}_{1}: \mu_{d} \neq 0 \\
& \text { em que } \mu_{d} \quad \text { é a média das diferenças } \\
& d_{i}=V_{\text {inglês }}-V_{\text {português. }}
\end{aligned}
$$

A reprodutibilidade entre as versões também foi avaliada usando coeficiente de correlação intraclasse (ICC). O ICC é definido pela razão entre a variância entre indivíduos e a variância total (Equação 1). Considerouse para ICC, segundo LANDIS \& KOCH (1977): concordância quase perfeita $(0,80-1,00)$, substancial $(0,60-0,79)$, moderada $(0,41-0,59)$, razoável $(0,21$ $0,40)$, discreta $(0,20-0)$ e pobre $(0-1)$.

$$
I C C=\frac{S_{b}^{2}}{S_{b}^{2}+S_{w}^{2}}
$$

Equação 1

em que $S_{b}^{2}$ é a variância entre indivíduos e $S_{w}^{2}$ é a variância dentre indivíduos.

Para o cálculo da confiabilidade do instrumento, utilizou-se o teste Alpha de Cronbach ( $\alpha$ ) (Equação 2). Para o Alpha de Cronbach, foram considerados ótimos os valores acima de 0,80 ; bons os valores acima de 0,70 e entre 0,60 e 0,70, nível aceitável (MONEY et al., 2005).

$$
\alpha=\frac{k}{k-1} \times\left(1-\frac{\sum_{j=1}^{k} S_{j}^{2}}{S_{T}^{2}}\right)
$$

Equação 2

em que $k$ é o número de itens do instrumento, $S_{j}^{2}$ é a variância do item $j(j=1, \ldots, k)$ e $S_{T}^{2}$ é a variância da soma das respostas de cada indivíduo. 
Tabela 1 - Versão original do instrumento de avaliação da neofobia em relação à tecnologia de alimentos sugerida por COX \& EVANS (2008): Food Technology Neophobia Scale.

\begin{tabular}{ll}
\hline Itens & \\
\hline 1 & New food technologies are something I am uncertain about. \\
2 & New foods are not healthier than traditional foods. \\
3 & The benefits of new food technologies are often grossly overstated. \\
4 & There are plenty of tasty foods around so we do not need to use new food technologies to produce more. \\
5 & New food technologies decrease the natural quality of food. \\
6 & New food technologies are unlikely to have long term negative health effects. * \\
7 & New food technologies give people more control over their food choices. * \\
8 & New products using new food technologies can help people have a balanced diet. * \\
9 & New food technologies may have long term negative environmental effects. \\
10 & It can be risky to switch to new food technologies too quickly. \\
11 & Society should not depend heavily on technologies to solve its food problems. \\
12 & There is no sense trying out high-tech food products because the ones I eat are already good enough. \\
13 & The media usually provides a balanced and unbiased view of new food technologies. * \\
\hline
\end{tabular}

* indicates reverse scored itens.

A classificação dos indivíduos quanto à neofobia em relação à tecnologia de alimentos é obtida pela soma dos valores individuais de cada item, que variam de 13 a 91 . O maior valor representa a menor receptividade dos consumidores para novas tecnologias (ou seja, maior neofobia) (COX \& EVANS, 2008). Para realização das análises estatísticas, as pontuações das questões $6,7,8$ e 13 foram invertidas, para que valores mais altos correspondam à maior neofobia. Para todas as análises, utilizou-se o programa SPSS (Social Package Statistical Science) versão 15.0, sendo considerados estatisticamente significativos valores de $\mathrm{P} \leq 0,05$.

\section{RESULTADOS E DISCUSSÃO}

A tradução e adaptação do questionário de Neofobia em relação à Tecnologia de Alimentos para a língua portuguesa foi simples, já que a versão original era de fácil compreensão e as escalas de medidas mantiveram-se as mesmas. Apenas o termo new food technologies, cuja tradução é novas tecnologias de alimentos, foi substituído por novas tecnologias empregadas na produção e/ou processamento de alimentos. De acordo com os tradutores bilingues, essa mudança facilita a compreensão pelos consumidores brasileiros dos itens que compõem o instrumento. A versão em português do questionário está apresentada na tabela 2, juntamente com a indicação dos itens que necessitam reverter as notas.

Em relação à reprodutividade, das 13 questões do instrumento avaliado, somente a questão 12 ("There is no sense trying out high-tech food products because the ones I eat are already good enough / Não faz sentido experimentar alimentos produzidos a partir de alta tecnologia, porque os que eu consumo já são bons o suficiente") apresentou diferença significativa entre as respostas da versão original e da versão traduzida para o português $(\mathrm{P}<0,05)$, pelo teste de Wilcoxon. No entanto, ao observar as respostas para a questão 12 , verificou-se que, dos 30 respondentes, sete responderam de forma idêntica as duas versões. No teste de Wilcoxon, as observações em que a diferença entre os pares é nula não são utilizadas para o cálculo da estatística. Esta limitação do teste pode ter contribuído para a não reprodutibilidade da questão 12 . Procedeu-se também à análise do coeficiente de correlação intraclasse (ICC) entre as versões inglesa e portuguesa para as 13 questões. Os resultados mostraram que todas as correlações foram significativamente maiores do que zero, variando entre 0,362 a $0,866(\mathrm{P}<0,05)$. A questão 12 apresentou o maior ICC, indicando alta correlação entre a versão original e traduzida, o que confirma sua reprodutibilidade. Para as demais questões, tanto para o teste de Wilcoxon como para o ICC, os resultados foram satisfatórios. O coeficiente de correlação intraclasse entre a soma total dos itens das duas versões foi de $\mathrm{ICC}=0,867(\mathrm{P}<0,05)$. Esse resultado é idêntico ao encontrado por EVANS et al. (2010) ao confirmar a confiabilidade do instrumento original Food Technology Neophobia Scale. A média, desvio padrão, mediana, teste de Wilcoxon, coeficientes de correlações intraclasse para as 13 questões das versões em inglês e em português estão apresentados na tabela 3 . 
Tabela 2 - Versão portuguesa do questionário Food Technology Neophobia Scale: Escala de Neofobia em relação à Tecnologia de Alimentos.

\begin{tabular}{|c|c|}
\hline \multicolumn{2}{|c|}{ Itens } \\
\hline 1 & $\begin{array}{l}\text { Eu não estou totalmente familiarizado com novas tecnologias empregadas na produção e/ou processamento de } \\
\text { alimentos. }\end{array}$ \\
\hline 2 & Novos alimentos não são mais saudáveis đo que os alimentos tradicionais. \\
\hline 3 & $\begin{array}{l}\text { As afirmações sobre os benefícios de novas tecnologias empregadas na produção e/ou processamento de alimentos são } \\
\text { frequentemente muito exageradas. }\end{array}$ \\
\hline 4 & $\begin{array}{l}\text { Já existem inúmeros alimentos saborosos no mercado, então nós não precisamos de novas tecnologias para produzir } \\
\text { mais alimentos. }\end{array}$ \\
\hline 5 & $\begin{array}{l}\text { Novas tecnologias empregadas na produção e/ou processamento de alimentos reduzem a qualidade natural dos } \\
\text { alimentos. }\end{array}$ \\
\hline 6 & $\begin{array}{l}\text { Novas tecnologias empregadas na produção e/ou processamento de alimentos provavelmente não trarão, a longo prazo, } \\
\text { efeitos negativos à saúde.* }\end{array}$ \\
\hline 7 & $\begin{array}{l}\text { Novas tecnologias empregadas na produção e/ou processamento de alimentos proporcionam às pessoas um maior } \\
\text { controle sobre as suas escolhas alimentares. }\end{array}$ \\
\hline 8 & Novos produtos que utilizam novas tecnologias de alimentos podem ajudar as pessoas a terem uma dieta equilibrada. $*$ \\
\hline 9 & $\begin{array}{l}\text { Novas tecnologias empregadas na produção e/ou processamento de alimentos podem causar, a longo prazo, efeitos } \\
\text { negativos ao meio ambiente. }\end{array}$ \\
\hline 10 & $\begin{array}{l}\text { Pode ser arriscado mudar rapidamente para novas tecnologias empregadas na produção e/ou processamento de } \\
\text { alimentos. }\end{array}$ \\
\hline 11 & A sociedade não deve depender demais de tecnologias para resolver os seus problemas alimentares. \\
\hline 12 & $\begin{array}{l}\text { Não faz sentido experimentar alimentos produzidos a partir de alta tecnologia, porque os que eu consumo já são bons o } \\
\text { suficiente. }\end{array}$ \\
\hline 13 & $\begin{array}{l}\text { A mídia geralmente fornece uma visão equilibrada e imparcial das novas tecnologias empregadas na produção e/ou } \\
\text { processamento de alimentos. * }\end{array}$ \\
\hline
\end{tabular}

* questões que necessitam reverter as notas.

Deve-se considerar ainda que não houve diferença estatística significativa entre a soma total dos itens das duas versões, pelo teste de Wilcoxon $(\mathrm{P}>0,05)$. Portanto, pode-se afirmar que a tradução do instrumento para o português foi conceitualmente equivalente à versão original.

Para medir a confiabilidade do instrumento, o coeficiente Alpha de Cronbach é o teste mais utilizado na literatura. De acordo com FACHEL \& CAMEY (2000), o objetivo da consistência interna é analisar se os itens possuem uma alta correlação. Considerando os 30 participantes, o coeficiente Alpha de Cronbach para as 13 questões do instrumento demonstrou alta confiabilidade $(\alpha=0,756)$. Procedeuse também à eliminação individual iterativa, a fim de validar a opção de manter todos os elementos do questionário e, analisando separadamente cada um dos itens, os valores de $\alpha$ se mantiveram entre 0,720 a 0,774 , refletindo um perfil de homogeneidade entre as variáveis analisadas. Portanto, pode-se afirmar que os itens são somáveis e constituem a representação de um mesmo construto.

Os resultados demonstram que a tradução do questionário de avaliação da neofobia em relação à tecnologia de alimentos para a língua portuguesa foi reprodutível e confiável, podendo ser empregada em estudos com consumidores usuários da língua portuguesa. A disponibilidade do presente instrumento em língua portuguesa é bastante útil para os profissionais da indústria de alimentos e pesquisadores durante o desenvolvimento de produtos utilizando novas tecnologias, uma vez que os consumidores neofóbicos podem ser identificados. Entender como o consumidor se comporta frente às novas tecnologias é interessante para elaboração de possíveis estratégias de marketing, a fim de aumentar a receptividade da população. De acordo com GRESHAM et al. (2006), aproximadamente 70 a $80 \%$ das inovações lançadas no mercado não são bem sucedidas. Dessa forma, fornecer informações aos consumidores sobre a tecnologia empregada na produção e/ou processamento do alimento pode aumentar a sua confiança frente ao novo produto.

\section{CONCLUSÃO}

A versão portuguesa do instrumento mostrou-se reprodutiva e confiável, o que permitirá identificar quais consumidores estão mais receosos ou mais receptíveis a aceitar uma nova tecnologia. Essa ferramenta poderá ser disponibilizada e utilizada em estudos com consumidores, quando se deseja avaliar a 
Tabela 3 - Média, desvio padrão, mediana, teste de Wilcoxon, coeficientes de correlações intraclasse (ICC) para as 13 questões das versões em inglês e em português ( $\mathrm{N}=30$ respondentes).

\begin{tabular}{|c|c|c|c|c|c|c|c|c|}
\hline \multirow[b]{2}{*}{ Variável } & \multicolumn{2}{|c|}{ 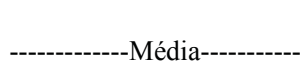 } & \multicolumn{2}{|c|}{------Desvio Padrão------ } & \multicolumn{2}{|c|}{---------Mediana--------- } & \multicolumn{2}{|c|}{$\begin{array}{c}\text { Teste de } \\
\text { Wilcoxon }\end{array}$} \\
\hline & Inglês & Português & Inglês & Português & Inglês & Português & $\mathrm{P}$ & ICC \\
\hline Par 1 (QUESTÃO 1) & 3,23 & 2,87 & 1,78 & 1,65 & 2,65 & 2,27 & 0,393 & 0,633 \\
\hline Par 2 (QUESTÃO 2) & 2,93 & 3,30 & 1,75 & 1,73 & 2,60 & 2,80 & 0,227 & 0,796 \\
\hline Par 3 (QUESTÃO 3) & 4,12 & 4,05 & 1,44 & 1,77 & 4,10 & 4,15 & 0,879 & 0,680 \\
\hline Par 4 (QUESTÃO 4) & 1,76 & 1,73 & 1,10 & 1,25 & 1,20 & 1,25 & 0,581 & 0,362 \\
\hline Par 5 (QUESTÃO 5) & 2,20 & 2,13 & 1,31 & 1,43 & 1,80 & 1,45 & 0,434 & 0,856 \\
\hline Par 6* (QUESTÃO 6) & 4,64 & 3,93 & 1,68 & 1,45 & 4,50 & 4,00 & 0,053 & 0,459 \\
\hline Par 7 * (QUESTÃO 7) & 3,41 & 3,11 & 1,68 & 1,77 & 3,00 & 2,50 & 0,234 & 0,431 \\
\hline Par 8* (QUESTÃO 8) & 2,57 & 2,63 & 1,37 & 1,31 & 2,35 & 2,45 & 0,857 & 0,746 \\
\hline Par 9 (QUESTÃO 9) & 3,50 & 3,80 & 1,92 & 1,76 & 2,95 & 4,10 & 0,227 & 0,796 \\
\hline Par 10 (QUESTÃO 10) & 3,69 & 4,35 & 1,85 & 1,90 & 3,98 & 4,86 & 0,064 & 0,693 \\
\hline Par 11 (QUESTÃO 11) & 3,77 & 3,56 & 1,81 & 1,67 & 3,88 & 3,35 & 0,387 & 0,620 \\
\hline Par 12 (QUESTÃO 12) & 1,99 & 1,62 & 1,24 & 0,92 & 1,70 & 1,30 & $0,010^{* *}$ & 0,866 \\
\hline Par 13 * (QUESTÃO 13) & 2,90 & 2,37 & 1,43 & 1,57 & 2,45 & 1,70 & 0,057 & 0,459 \\
\hline Par 14 (Soma das 13 questões) & 43,48 & 44,10 & 10,05 & 10,04 & 41,50 & 42,49 & 0,586 & 0,867 \\
\hline
\end{tabular}

* questões em que as notas foram revertidas. ${ }^{* *}$ significativo ao nível de $5 \%$ de probabilidade.

atitude frente às tecnologias empregadas na produção e/ou processamento de alimentos, prevendo, assim, a disposição dos indivíduos em experimentar alimentos produzidos por novas tecnologias.

\section{AGRADECIMENTOS}

Á Coordenação de Aperfeiçoamento de Pessoal de Nível Superior (CAPES), ao Conselho Nacional de Desenvolvimento Científico e Tecnológico (CNPq) e a Fundação de Amparo à Pesquisa do Estado de Minas Gerais. (FAPEMIG).

\section{COMITÊ DE ÉTICA}

Este projeto foi aprovado pela Comissão de PósGraduação do Departamento de Tecnologia de Alimentos - Universidade Federal de Viçosa - UFV, registro número $50717262567 / 2012$, obedecendo, conforme descrito, os requisitos necessários para sua publicação.

\section{REFERÊNCIAS}

BARRENA, R.; SÁNCHEZ, M. Neophobia, personal consumer values and novel food acceptance. Food Quality and Preference, v.27, n.1, p.72-84, 2012. Disponível em: <http:// dx.doi. org/10.1016/j.foodqual.2012.06.007>. Acesso em: 15 out. 2012.

BEHRENS, J.H. et al. Brazilian consumer views on food irradiation. Innovative Food Science and Emerging Technologies, v.10, p.383-389, 2009. Disponível em: <http://dx. doi.org/10.1016/j. ifset.2009.01.001>. Acesso em: 13 out. 2012.

BIEBERSTEIN, A. et al. Consumer choices for nano-food and nano-packaging in France and Germany. European Review of Agricultural Economics, v.40, n.4, p.73-94, 2013. Disponível em: <http://erae.oxfordjournals.org/content/40/1/73>. Acesso em: 20 mai. 2013.

CARDELLO, A.V. Consumer concerns and expectations about novel food processing technologies: Effects on product liking. Appetite, v. 40, p. $217-233,2003$.

CHEN, Q. et al. Measuring consumer resistance to a new food technology: a choice experiment in meat packaging. Food Quality and Preference, v. 28, n. 2, 2013. Disponível em: <http://dx.doi. org/10.1016/j.foodqual.2012.10.008>. Acesso em: 10 mai. 2013.

CORSO, M.P.; BENASSI, M.T. Tradução e validação para a língua portuguesa de questionário sobre fatores cognitivos e comportamentais que afetam a aceitação de alimentos funcionais. Alimentos e Nutrição, v. 23, p. 65-70, 2012. Disponível em: $<$ http://serv-bib.fcfar.unesp.br/seer/index.php/alimentos/ article/viewFile/1825/1825>. Acesso em: 14 out. 2012.

COX, D.N.; EVANS, G. Construction and validation of a psychometric scale to measure consumers' fears of novel food technologies: the Food Technology Neophobia Scale. Food Quality \& Preference, v. 1, p. 704-710, 2008.

DANTAS, M.I.S. et al. Tradução e validação para a língua portuguesa do questionário Health Consciousness utilizado em estudos de consumidor. Boletim Sociedade Brasileira de Ciência e Tecnologia de Alimentos, v.37, n.2, p.103-105, 2003.

DELIZA, R. et al. Consumer attitude towards information on non conventional technology. Trends in Food Science \& Technology, v.14, p.43-49, 2003a. Disponível em: <http://dx. doi.org/10.1016/ S0924-2244(02)00240-6>. Acesso em: 14 out. 2012.

DELIZA, R. et al. Tradução e validação para a língua portuguesa de questionário utilizado em estudos de consumidor. Ciência e Tecnologia de Alimentos, v. 23, n. 1, p. 43-48, 2003 b. 
EVANS, G.; COX, D.N. Australian consumers' antecedents of attitudes towards foods produced by novel technologies. British Food Journal, v.108, n.11, p.916-930, 2006.

EVANS, G. et al. Reliability and predictive validity of the Food Technology Neophobia Scale. Appetite, v.54, p.390-393, 2010. Disponível em: <http://dx.doi.org/10.1016/ j.appet.2009.11.014>. Acesso em: 12 out. 2012. doi: 10.1016/j.appet.2009.11.014.

FACHEL, J.M.G.; CAMEY, S. Avaliação psicométrica: a qualidade das medidas e o entendimento dos dados. In: CUNHA, J. A. Psicodiagnóstico. Porto Alegre: Artes Médicas, 2000. p. 158-170.

GRESHAM, G. et al. Inter-functional market orientation between marketing departments and technical departments in the management of the new product development process. Journal of Behavioral and Applied Management, v. 8, n. 1, p. 53-55, 2006. Disponível em: <http:// www.ibam.com/pubs/jbam/articles/vol8/ no1/JBAM_8_1_3.pdf>. Acesso em: 20 out. 2012.

HANSEN, J. et al. Beyond the knowledge deficit: Recent research into lay and expert attitudes to food risk. Appetite, v.41, p.111$121,2003$.

LANDIS, J.R.; KOCH, G.G. The measurement of observer agreement for categorical data. Biometrics, v.33, p.159-174, 1977. Disponível em: <http://www.jstor.org/stable/2529310>. Acesso em: 14 out. 2012.

MONEY, A. et al. Fundamentos de métodos de pesquisa em administração. Porto Alegre: Bookman Companhia, 2005. 472p.

OHRBACH, R. et al. Guidelines for establishing cultural equivalency of instruments. New York: University at Buffalo, 2009. 59 p.

PETER, J.P. Reliability: a review of psychometric basics and recent marketing practices. Journal of Marketing Research, v.16, n.1, p.6-17, 1979. Disponível em: <http://www.jstor. org/ stable/3150868>.Acesso em: 13 out. 2012.
PLINER, P.; HOBDEN, K. Development of a scale to measure the trait of food neophobia in humans. Appetite, v.19, p.105-120, 1992.

PRIETO, A.J. A method for translation of instruments to other languages. Adult Education Quarterly, v.43, n.1, p.1-14, 1992.

RONTELTAP, A. et al. Consumer acceptance of technology based food innovations: lessons for the future of nutrigenomics. Appetite,v.49, p.1-17, 2007.

SANGUANSRI, P., AUGUSTIN, M.A. Nanoscale materials development - a food industry perspective. Trends in Food Science \& Technology, v.17, p.547-556, 2006. Disponível em: $<$ http://dx.doi.org/10.1016/j.tifs.2006.04.010>. Acesso em: 13 out. 2012 .

SIEGRIST, M. Factors influencing public acceptance of innovative food technologies and products. Trends in Food Science \& Technology, v.19, p.603-608, 2008. Disponível em: $<$ http://dx.doi.org/10.1016/j.tifs.2008.01.017 >. Acesso em: 14 out. 2012.

SOARES, L.L.S. et al. Escalas atitudinais utilizadas em estudos de consumidor: tradução e validação para a língua portuguesa. Alimentos e Nutrição,v.17,p.51-64, 2006. Disponível em: $<$ http://200.145.71.150/seer/index.php/alimentos/article/ view/211/213>. Acesso em:9out.2012.

van KLEEF, E. et al. Consumer research in the early stages of new product development: A critical review of methods and techniques. Food Quality \& Preference, v. 16, p.181-201, 2005.

VIDIGAL, M.C.T.R. et al. Effect of a health claim on consumer acceptance of exotic Brazilian fruit juices: Açaí (Euterpe oleracea Mart.), Camu-camu (Myrciaria dubia), Cajá (Spondias lutea L.) and Umbu (Spondias tuberosa Arruda). Food Research International, v.44, p.1988-1996, 2011. 